\title{
SURVEILLANCE AND TRACKING OF ELEPHANTS USING VOCAL SPECTRAL INFORMATION
}

\author{
J.Nirmal Prince ${ }^{1}$, S.J.Sugumar ${ }^{2}$ \\ ${ }^{1}$ Department of Electrical and Electronics Engineering, Coimbatore Institute of Technology, Coimbatore, India \\ ${ }^{2}$ Department of Electrical and Electronics Engineering, Coimbatore Institute of Technology, Coimbatore, India
}

\begin{abstract}
Human-elephant conflict is a major problem which leads to crop damage, human death and injuries caused by elephants, and elephants being killed by humans. Elephant Intrusion has been on the rise in the forest border areas with herds of elephants straying into human habitation and creating a great loss to their properties. The surveillance and tracking of elephants are difficult due to their size and nature of movement. In this paper, a method for detecting and tracking elephants along the forest border areas using the vocal communications of elephants is presented. Two approaches are used one is to find the spectral energy magnitude and the other to determine highest pitch frequency produced by elephants. A threshold is identified for the two approaches; once the elephant vocal communication signal crosses the limit an alert will be sent to the forest officials notifying them about the elephant intrusion to redirect the elephant back to the forest.
\end{abstract}

Keywords-elephant vocalization, human elephant conflict; spectral energy ; pitch frequency

*** $*$

\section{INTRODUCTION}

The Asian elephant (Elephasmaximus) is highly threatened by habitat fragmentation, habitat loss and human-elephant conflict. India hosts $60 \%$ of Asian elephant population, nearly two-thirds of the elephant population lives either close to or within humandominated landscapes. Southern India harbors half of India's elephant population containing about 6300 elephants [1]. The increase in human population in India propelled by agricultural and industrial growth has led to the conversion of the forest lands into human settlements. Due to this, the wild elephant and other animal population face acute shortage of resources such as water and food, making them move often into the human habitat. Hence there has been severe man- elephant conflict. The conflict has been on the rise in the forest border areas with herds of wild pachyderms straying into human habitation. The surveillance and tracking of these herds are difficult due to their size and nature of movement. The time to recover from the danger is negligible; hence the loss due to destruction in the farms is more. The elephants are also subject to attack by humans resulting in danger to the life of elephants. According to the authors in [2], poaching for ivory had indeed become a threat with 100-150 tuskers being lost annually to illegal killings.

Human-elephant conflict (HEC) is a key example of the growing competition between people and wildlife for space and resources throughout Africa and Asia. This study explores the correlation of reported HEC incidents within 58 villages between $80 \mathrm{~km}$ from the boundary of Kallar to walayar, Coimbatore, Tamil Nadu, India. Habitat loss and fragmentation is the biggest threat to the continuing survival of Asian elephants in this region. As the human population has inexorably risen, the forest wild lands in which elephants live have been disappearing. Human elephant conflict is on the rise and it is a battle that the elephant is losing. As elephant habitat diminishes, the elephants are pushed into increasingly smaller areas. The serious consequence of the shortage of wild food leads to a corresponding increase of crop raiding and incidents of human-elephant conflict [2].

\section{RELATED WORK}

Many methods are followed to avoid HEC: Construction of elephant proof trenches, solar electric fencing, beehives to mitigate elephant crop depredation. Satellite technology with elephants tagged with radio collars. In [3] Seneviratne, et.aldiscusses the design and implementation of an electronic sensor and analysis system for the detection of the said Infrasound elephant calls. In [4] G.D. De Silva andKdeZoysa, designed an infrasound recording system (hardware and software consists of a speaker (infrasound sensor) as an analog input, an amplifier, an anti-aliasing filter, an analog to digital (A/D) converter and finally a PC application which is used to analyze the recorded signal. In [5] Venter,P.J., and Hanekom,J.J proposed the possibility of using the elephant-elephant communication (elephant rumbles) to detect the presence of a herd of elephants in close proximity, In this work the authors have recorded the low frequency infrasound pattern but they do not compare with that of other animals to confirm an elephant occurrence. In the above methods audio features of elephants were used to detection. They have only tried with infrasonic calls of elephants moreover in forest border areas other animal sounds are also available in the above works they have not 
shown results on discriminating the elephant sounds from other sounds. In this research we explore the prospect of using elephant vocalizations for detecting crop raiding elephants before they enter human living areas. We also compare the sounds of elephants with that of other species and explore the opportunity of detecting elephants only.

It is easier to chase elephants before they enter fields and therefore, most damage can be averted [2]. An early warning system to minimize the human-elephant conflict in the forest border areas using elephant vocalization is proposed in this article. The system helps mitigate such conflicts in two ways: Provides warning to people about the anticipated entry of elephants into human habitation. Provides advance information to the authorities to take action to chase the pachyderms back to the forest.

\section{MATERIALS AND METHODS}

In the first of our previous two works we used seismic sensors to detect the movement of elephants in forest border areas [6], whenever the elephant walk across the region of seismic sensors an alert is sent to forest officials and in our second work we used image cameras to capture the images and compare with a data base to detect the movement of elephants [7].To explore the area of elephant vocalization, In this research work the recordings of elephant call sounds are used to determine the movement of elephants in the forest border areas.

\subsection{Study Area}

Studies were made in the Coimbatore Forest Division, Tamil Nadu, India. The data were collected from the Coimbatore Forest Department website, interviews with village people affected by elephants and relevant literature. Coimbatore district is richly endowed with hills, forests, rivers and wildlife. Geographical area of the district is $74,433.72$ sq. $\mathrm{km}$ with a forest area of 693.48 sq. $\mathrm{km}(9.33 \%)$ [16]. The number of incidents of elephants straying into farm lands was 680 in 2011, 844 in 2010 and 560 in 2009 . The number of people killed in elephant attacks in Coimbatore was 13 in 2012, 8 in 2011, 15 in 2010 and 11 in 2009. The number of elephants killed by such conflicts was 4 in 2012, 1 in 2011, 1 in 2010 and 2 in 2009 (Courtesy: The HINDU, COIMBATORE, February 5, 2013).

\subsection{Background}

Elephants are the largest terrestrial mammals which uses vocalizations to communicate over short and long distances. The elephant vocalization in the frequency contains abundance of information about elephant social living. Some of the practical issues include the difficulty of acquiring continuous data from forest environments and identifying elephant signals within recordings are resolved. Asian elephant calls can be categorized in to 4 basic types they are Trumpet, Roar, Chirp, and Rumble [8]. The call type and its frequency range used by elephants are given in Table 1. Elephant call signal frequency varies from $5 \mathrm{~Hz}$ to $10,000 \mathrm{~Hz}$ which includes harmonics also. Out of the 258 measurable calls described by [8], close to $30 \%$ are trumpets, $22 \%$ are roars, 26\% are 'chirps' (squeaks) and $22 \%$ are rumbles.

Table 1: Call Type Frequency Of Asian Elephants

\begin{tabular}{|l|l|}
\hline Call Type & Frequency range(Hz) \\
\hline Trumpet & $405-5879$ \\
\hline Roar & $305-6150$ \\
\hline Chirp (Squeak/Squeal) & $313-3370$ \\
\hline Rumble & $10-173$ \\
\hline
\end{tabular}

Elephant uses calls in the context of play, disturbances, presence of other species or vehicles, interactions within herds, during encounters with other species. Rumbling is used to inform let's go, contact calling, and greeting. Elephant vocalization can be established with amplitudes of $112 \mathrm{~dB}$ at 1 $\mathrm{m}$ and $46 \mathrm{~dB}$ at 2,048 $\mathrm{m} \mathrm{[15].}$

\subsection{Hardware Model}

Obtaining high quality, continuous recordings of elephant vocalizations in the wild is a difficult task. The recordings are done via an FM system unit which contains a FM transmitter and receiver at the base station. The audio transmitter is placed in certain pockets where elephants enter into human living areas from the forest. The FM transmitter is a low-power battery driven; the device is tuned in such a way that it has an average range up to about 5 to 20 meters with a frequency range of 102.3 MHz The environmental signal is captured via an audio mike, which converts any audio signal in to electrical variations and is amplified with gain of 20 times the input signal. Sources of noise like wind and vehicle were noted for filtering during data analysis. The audio input is given to PC via an audio jack for elephant vocalization analysis. A custom-written program in Matlab is used to detect the audio features of elephant calls.

\subsection{Elephant Vocal Detection System}

Two approaches are employed for the detection of elephant vocal communication in the forest border areas as shown in Fig 1 .

1) Estimation of Maximum and Standard Deviation of Elephant Call Frequency

2) Elephant Call Pitch Determination 


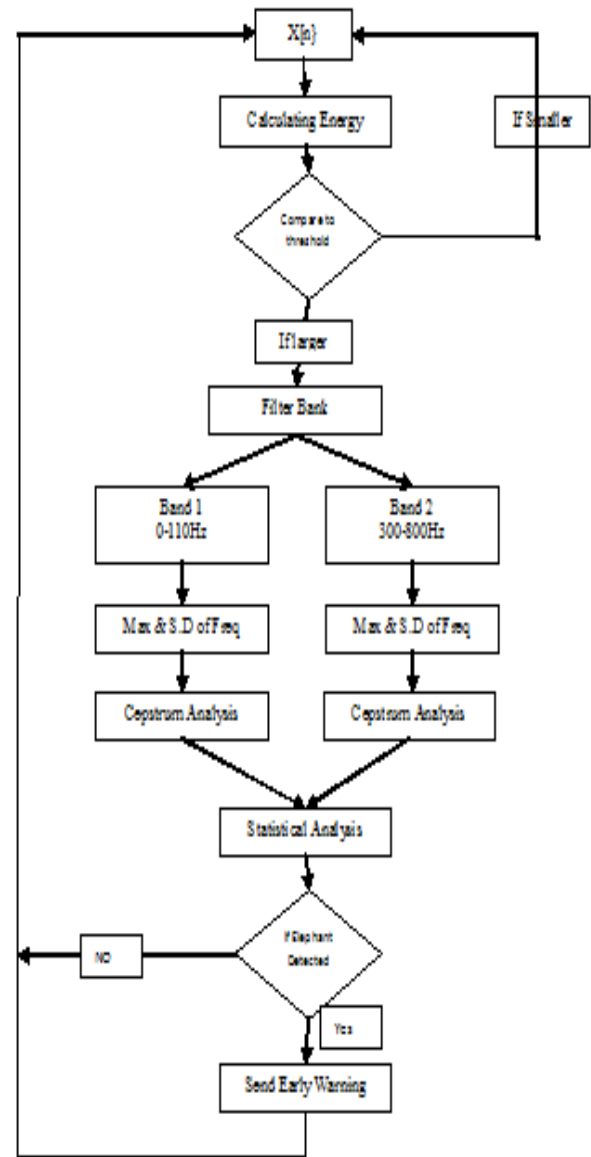

Fig. 1 Flowchart of the Elephant Detection System

In the first method, the received elephant sound signals are recorded as a .wav file in the PC and updated in a directory every 5seconds; the recorded signal sample is shown in Fig 2. The recorded signal is filtered with the hamming filter with the window size of $20(\mathrm{~ms})$ then Modified Periodogram Power Spectral Density Estimate is calculated using Periodogram approach then the noise signals are zero padded, the maximum of the noise padded signals is the maximum frequency and the vocalizations are applied for Cepestral Pitch Determination The lowest frequency of the autocorrelation of each channel gives an indication of the fundamental frequency component present in that channel for the particular segment. We acquire an estimate of the fundamental frequency of the signal using frequency domain cepstral approach. This is used primarily to determine if the received signal is elephant or non-elephant using the fundamental frequency. By determining the maximum, minimum and fundamental frequencies an early warning is sent to the forest officials to take necessary actions on approaching elephant.

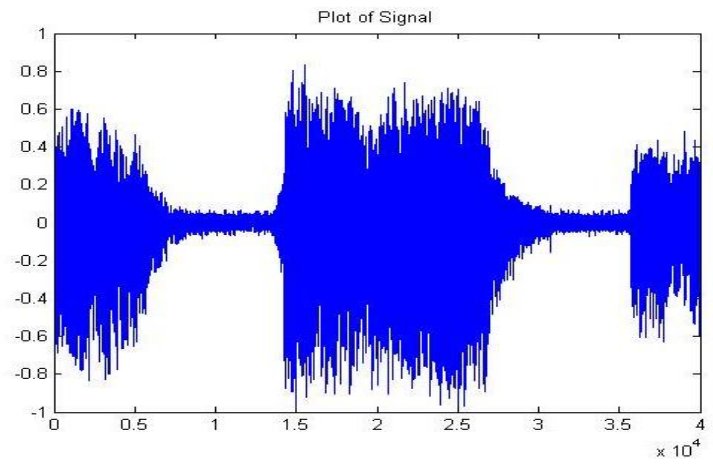

Fig. 2.Elephant Sound Recorded Sample

\section{PREPROCESSING}

The main objective of the work is to discriminate elephant sound signal from that of other animals in the presence of forest noises such as sounds of birds, insects, trees and wind. The best-known filter approximation is the Butterworth or maximally-flat response. It exhibits a nearly flat pass band with no ripple. The Butterworth filter of order $\mathrm{n}$ is described by the magnitude squared of its frequency response

$$
[H(\omega)]^{2}=\frac{1}{1+\left(\frac{\omega}{\omega_{0}}\right)^{2 n}}
$$

Where $\mathrm{n}$ is the order of the filter, and can be any positive whole number $(1,2,3, \ldots)$, and $\omega_{0}$ is the $-3 \mathrm{~dB}$ frequency of the filter [9]. The transfer function of $H_{8}(s)$ for the normalized [4] Butterworth filter of order 8is

$H_{8}(s)=\frac{1}{\left(s^{2}+0.3902 s+1\right)\left(s^{2}+1.111 s+1\right)\left(s^{2}+1.6629 s+1\right)\left(s^{2}+1.8794 s+1\right)}(2)$

Let $H_{L P}(s)$ Represents a low pass filter with cutoff frequency of $\omega_{\mathrm{c}}$ and the transfer function is given by $\mathrm{s} \rightarrow \mathrm{s} / \omega_{\mathrm{c}}$. The bandpass filter with transfer function $H_{B P}(s)$ is given by

$H_{B P}(s)=H_{L P}(s) \mid s \rightarrow\left(s^{2}+\omega_{l} \omega_{u}\right) /\left[s\left(\omega_{u}-\omega_{l}\right)\right](3)$

Where $\omega_{1}$ and $\omega_{2}$ are the frequency beyond $\omega_{l}$ and $\omega_{u} . \omega_{l}$ and $\omega_{u}$ are the lower and upper cutoff frequencies for the bandpass filter.

\section{SPECTROGRAM ANALYSIS}

A spectrogram is a representation of how the frequency content of a signal changes with time. The spectrogram is the result of calculating the frequency spectrum of windowed frames of a compound signal. It is a three-dimensional plot of the energy of the frequency content of a signal as it changes over time. Spectrograms are used to identify phonetic sounds, to analyze the cries of animals, and in the fields of music, sonar/radar, speech processing, etc. Spectrograms are usually calculated from the time signal using the short-time Fourier transform (STFT). It is a Fourier-related transform used to determine the 
sinusoidal frequency and phase content of local sections of a signal as it changes over time and is given as.

$\operatorname{STFT}\{x[n]\}=\sum_{n=-\infty}^{\infty} x[n] \omega[n-m] e^{-i \omega n}$

Spectrograms of these signals (DFT with Hamming window size of $5 \mathrm{~ms}, 50 \%$ overlap) were used for further analysis.

\section{ESTIMATION OF MAXIMUM AND STANDARD DEVIATION OF ELEPHANT CALL FREQUENCY}

A narrow-band spectrogram was computed and the minimum and standard deviation of frequencies of the elephant calls were determined using their power spectral densities. To estimate the essential bandwidth, the power spectral density (PSD) of the noisy signal was first computed using a Hamming window (20 $\mathrm{ms})$. The PSD of the noise was then estimated using the initial 5 $\mathrm{s}$ of the recording. The estimated PSD of the noise was subtracted from that of the noisy signal to yield an estimate of the PSD of the signal. In general, the estimate had positive values at all discrete Fourier transforms (DFT) bins; any negative values due to estimation errors were clamped to zero. From this estimate, the bandpass region containing about $80 \%$ of the total energy was considered as the frequency range of the signal. For rumbles, however, calls were first low-pass-filtered with a cutoff of 5 to $110 \mathrm{~Hz}$ (this value was based on a preliminary inspection of the spectrograms). The signals thus obtained were re-sampled (by the zero padding approach) to obtain more points along the frequency axis.

\section{ELEPHANT VOCAL CALL PITCHFREQUENCY DETERMINATION}

Natural sounds are a composition of a fundamental frequency with a set of harmonics which occur at near integer multiples of that fundamental. The frequency that interprets as the pitch of a sound is this fundamental frequency [11].Pitch is largely responsible for inflections in elephant calls used for communicating and expressing itself. These inflections also play a role in allowing us to consistently identify an elephant. Pitch is a perceptive quality that describes the highness or lowness of a sound. For a harmonic signal this is the lowest frequency in the harmonic series. The fundamental frequency can be deduced from the other frequency components provided that they are integer multiples of F0 [12]. This work attempts to develop a pitch tracking and detecting method to sense the movement of elephants in the forest border areas. The pitch determination is very important for many speech processing algorithms. In this work, pitch detection methods via cepstrum method is proposed. Cepstral analysis provides a way for the estimation of pitch [13]. If we assume that a sequence of an elephant vocal is the result of convoluting the glottal excitation sequence e[n] with the vocal tract's discrete impulse response $\theta[\mathrm{n}]$. In frequency domain, the convolution relationship becomes a multiplication relationship. Then, using property of $\log$ function $\log \mathrm{AB}=\log \mathrm{A}+\log \mathrm{B}$, the multiplication relationship can be transformed into an additive relationship. Finally, the real cepstrum of a signal $s[n]=e[n]+\theta[n]$ is defined as

$c[n]=\frac{1}{2 \pi} \int_{-\pi}^{\pi} \log |s(w)| e^{j n w} d w$

WhereS $(\omega)=\sum_{n=-i n f}^{\text {inf }} s[n] e^{-j w n}$

Cepstrum is a Fourier analysis of the logarithmic amplitude spectrum of the signal. If the log amplitude spectrum contains many regularly spaced harmonics, then the Fourier analysis of the spectrum will show a peak corresponding to the spacing between the harmonics: i.e. the fundamental frequency. Effectively we are treating the signal spectrum as another signal, and then looking for periodicity in the spectrum itself.

\section{RESULTS AND DISCUSSION}

\subsection{Field Observations}

The field observations are carried out in the forest border areas in Mettupalayam elephant camp where 36 temple elephants and 18 kumki elephants were trained during the period of December 2013 to February 2014 is shown in Fig 3.

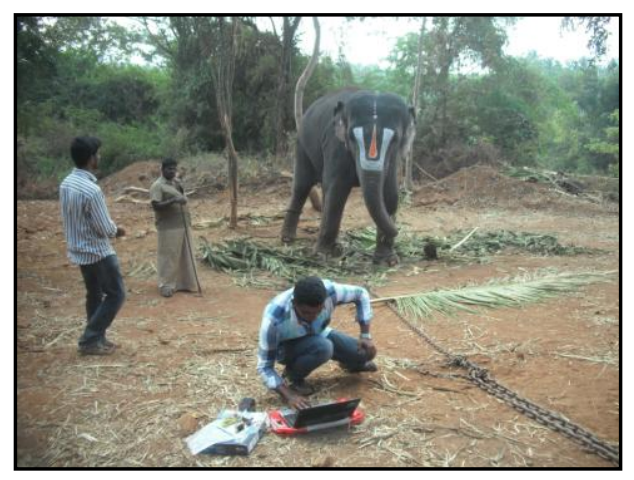

Fig.3. Field Testing

In comparison with existing work done in [3], [4] and [5], where the authors present only elephant detection scheme and they do not discriminate elephant signal from other sound signals in the forest environment. In this work we have devised an algorithm which explores the spectral information of sound produced by animals to discriminate elephants from other animals. We recorded the vocalization produced by three different Asian elephant's one male and two female in the elephant camp. For analysis about the different elephant call type we used the recorded elephant audio samples from the web site [15].The received input audio signal is first filtered by an $8^{\text {th }}$ order low pass filter to obtain infrasonic spectrum for detection of low frequency rumbles, the low passfilter spectrum of an elephant rumble in the range of $150 \mathrm{~Hz}$. The same audio input is given toan $8^{\text {th }}$ order Butterworth bandpass filter to obtain 
spectrum of other elephant vocal signals in the region of 400 to $800 \mathrm{~Hz}$ the acquired filter spectrum of an elephant trumpet is shown in Fig.4.

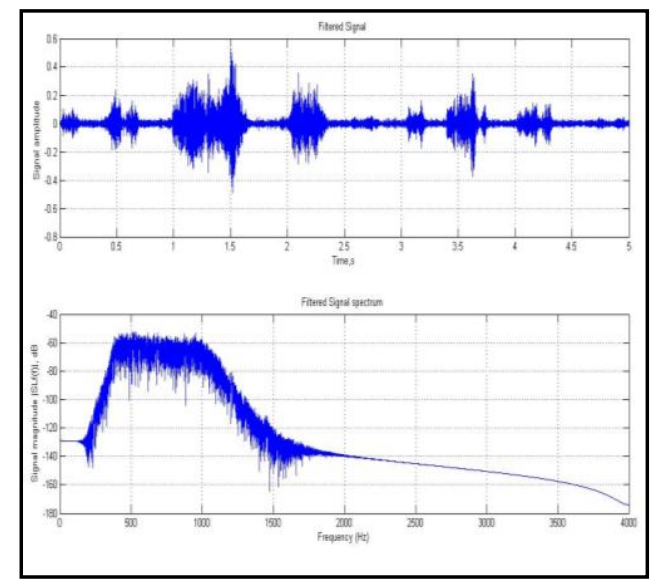

Fig.4. Frequency Spectrum of a Filtered Trumpet

\subsection{Spectrogram Analysis}

In this work we have analyzed elephant calls by visualizing using spectrogram. Fig 5 shows a spectrogram of a typical elephant rumble. The spectrogram obtained at $7 \mathrm{KHz}$ of rumble frequency.12 harmonics of the rumble occurring from $0.4 \mathrm{~s}$ to $2.7 \mathrm{~s}$ is produced by the elephant. Harmonics appearing around $0.4 \mathrm{~s}$ to $2.7 \mathrm{~s}$ for the $1^{\text {st }}$ rumble and from $3 \mathrm{~s}$ to $5.2 \mathrm{~s}$ for the $2^{\text {nd }}$ rumble. The spectrogram appears after $5.5 \mathrm{~s}$ and up to $10.5 \mathrm{~s}$ is the mirror image of the spectrogram.

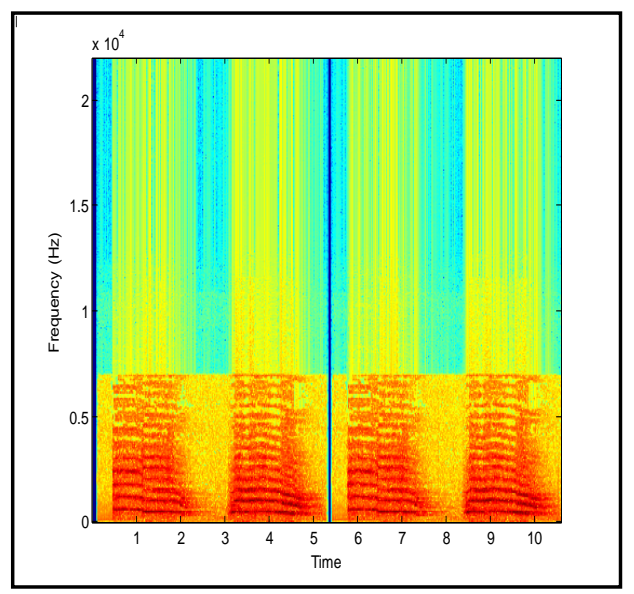

Fig.5. Spectrum of a Trumpet

Fig.6 shows a spectrogram for a Monkey signal. The spectrogram obtained at $18 \mathrm{KHz}$ of rumble frequency. More harmonics occurred and each spectral content occurred for short period of time. The largest time of signal identified is only of 0.6 seconds.

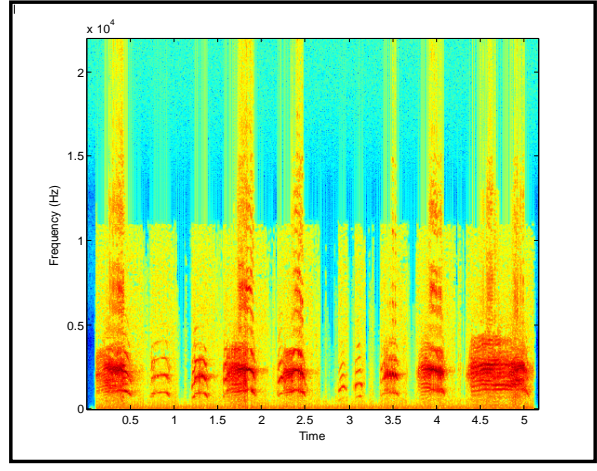

Fig.6. Spectrogram of a Monkey

\subsection{Estimation of Maximum and Minimum}

\section{Frequencies}

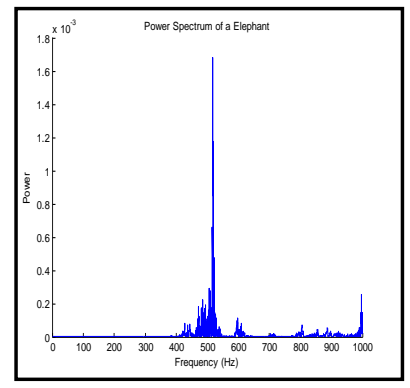

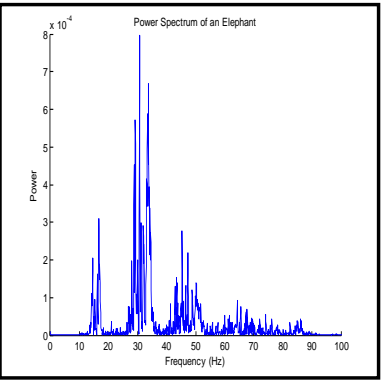

$\mathrm{b}$
Fig.7 a Power Spectral Density Fig 7 b Power Spectral Density of an Elephant Trumpet of Elephant Rumble

The power spectrum of the elephant trumpet and rumble signal seen in Fig.7Adepicts that the dominant frequency components of the signal lie in the range of $400 \mathrm{~Hz}$ to $1000 \mathrm{~Hz}$ with its peak at $503 \mathrm{~Hz}$ at $1.8 \mathrm{mw}$ of power. The power spectrum of the elephant rumble is shown in Fig.7B which depicts that the dominant frequency components of the signal lie in the range of 10 to $90 \mathrm{~Hz}$ with its peak at $32.0029 \mathrm{~Hz}$ at $0.8 \mathrm{mw}$ of power. The maximum frequency of the test species is obtained and normalized and depicted in Fig 8.

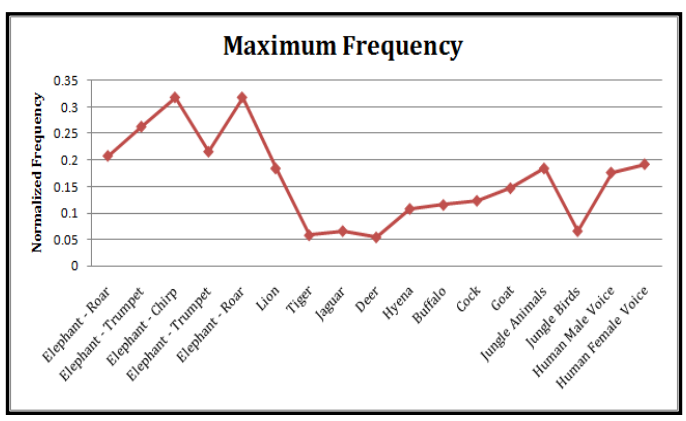

Fig.8 Estimated maximum Frequency of Various Species 
We also determined the standard deviation of frequency contents of all the test species sound signals and the results are depicted in Fig 9.

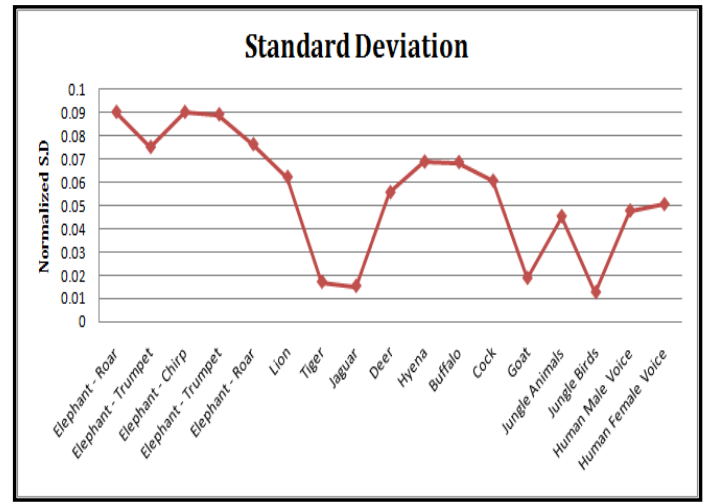

Fig.9. Standard Deviation of Various Species

\subsection{Pitch Frequency Determination}

This work attempts to develop a pitch tracking and detecting method to sense the movement of elephants in the forest border areas. The elephant vocal signals samples were given to the software developed which estimates the pitch frequency. The cepstrum is so-called because it turns the spectrum inside-out. The $\mathrm{x}$-axis of the cepstrum has units of frequency, and peaks in the cepstrum (which relate to periodicities in the spectrum) are called harmonics. To obtain an estimate of the fundamental frequency from the cepstrum we look for a peak in the frequency region corresponding to typical speech fundamental frequencies (1/frequency).The pitch estimates are calculated from the fundamental frequency in each of the Cepstral space. An elephant trumpet of duration 2 seconds was given to the pitch tracker which detected at $1.1726 \mathrm{~s}$ with highest pitch tracked at frequency $41.4827 \mathrm{~Hz}$ as shown in Fig 10 .
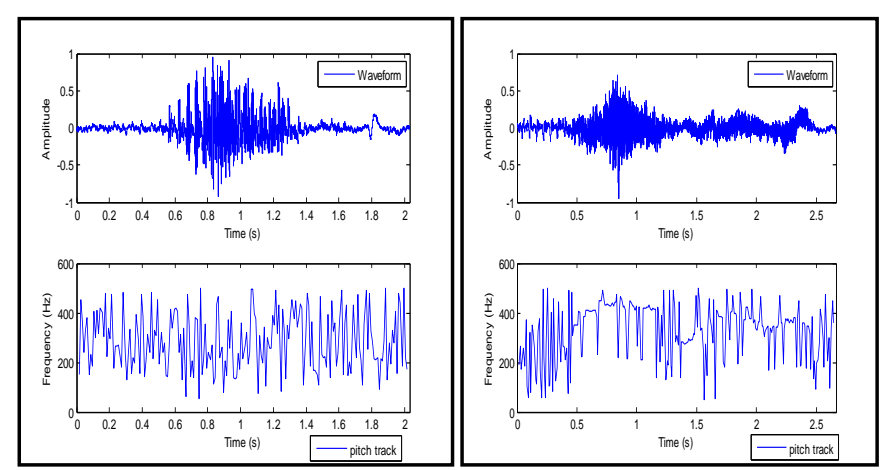

Fig.10. Pitch of Elephant Rumble Fig.11.Pitch of Elephant Trumpet

An elephant rumble of duration 2.5 seconds was given to the pitch tracker and the pitch occurred at $500.3314 \mathrm{~Hz}$ at $1.1319 \mathrm{sas}$ shown in Fig10.

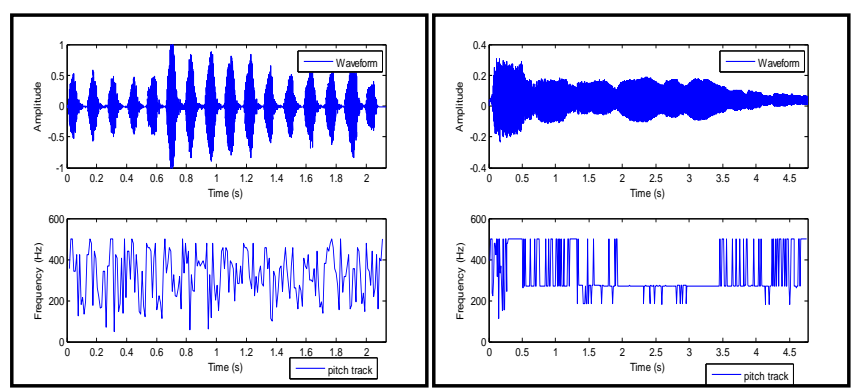

Fig.12. Pitch of Bird Chirp Fig: 13 Pitch Track of Wolf

The pitch frequencies were recorded for animals which are common in forest border areas and are shown in Fig. 12 \& 13. Comparison of pitch frequencies of elephants and other animals are done. However, most animal pitch frequencies fall below $400 \mathrm{~Hz}$. But sounds of birds and monkeys were found higher than elephant pitch frequencies. And also weak elephant rumbles were not detected and false alarms increased. So it was a challenge in discriminating the elephant signals and other animal sounds. From the above two methods we decided to use both peak spectral magnitude and peak frequency as combined parameters to discriminate elephant sound signal from that of other animals in the presence of forest noises such as sounds of birds, insects, trees and wind. The peak spectral magnitude was set to 1.0 and pitch frequency set to $400 \mathrm{~Hz}$ to $800 \mathrm{~Hz}$ bandwidth for detecting elephants. The algorithm developed such that it also able to detect low frequency rumbles in the range of 20 to $50 \mathrm{~Hz}$. All these signals contain the fundamental frequency and the harmonics. We tested the system in Mettupalayam elephant camp, Coimbatore, India; the system detected only the elephant sound signal.

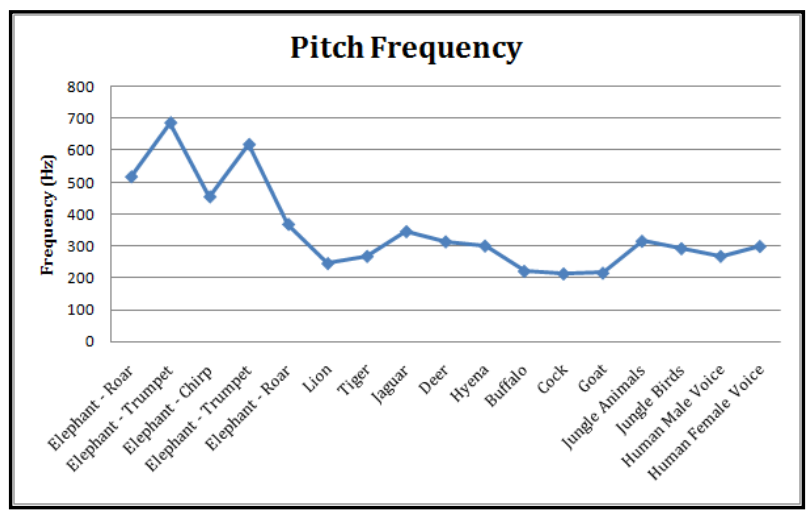

Fig.14 PitchFrequency Comparison for Varipus Species

A GUI has been developed for the elephant detection system and is shown in Fig 15. This is an automated system which records audio signal for every 5 secs and test for elephant signal. Once elephant signal is detected and an early warning in form of GSM message is sent to forest officials. 


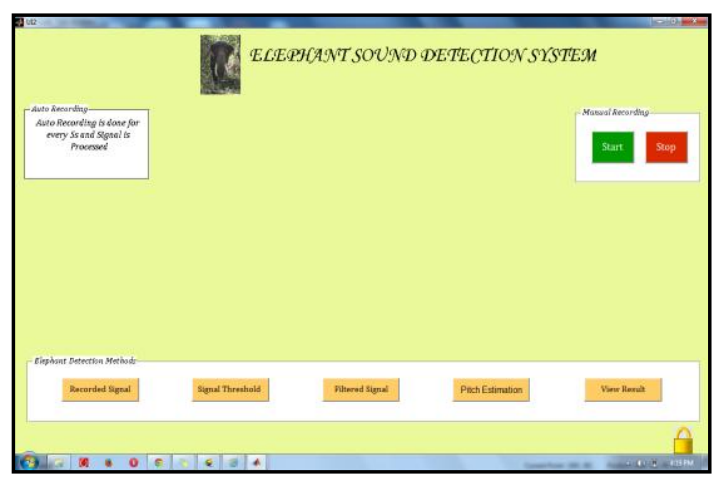

Fig.15 GUI for Elephant Vocal Sound Detection System

\section{CONCLUSIONS}

Elephants use vocalizations for both long and short distance communication. In this work, the vocal repertoire of wild Asian elephants in southern India was examined. Elephants produce four types of calls namely, trumpets, chirps, roars, and rumbles, based on quantitative analyses of their spectral and temporal features. These calls were made by elephants in the context of play, distress, disturbance, aggression, confusion, alarm and contact calling within and among herds. The spectral and temporal features of the four call types were used as a feature for detecting elephant even in the presence of other animals and noised present in the forest. Two spectral features spectral energy thresholding and pitch frequency detection were used in this work in discriminating and detecting the presence of elephant in human habitat areas. The analysis provides insights to protect elephants from human activities and reduces the work effort of forest officials and provides solution for human elephant conflict. The system is completely automated; the strength of this approach stems from the ability to discriminate elephants which is the key in avoiding false alarm.

\section{ACKNOWLEDGMENTS}

We thank the Coimbatore forest department for providing permission to take sample readings from elephants in the Mettupalayam elephant camp, Coimbatore.

\section{REFERENCES}

[1] Kumara.H.N ,Rathnakumar.S, Ananda Kumar M \&Singh M. (2012). Estimating Asian elephant,Elephasmaximus, density through distance sampling in the tropical forests of BiligiriRangaswamy Temple Tiger Reserve, India. Journal - Tropical Conservation Science. 5(2) .163-172.

[2] Lenin, J. and Sukumar,R. 2011. Action Plan for the Mitigation of Elepant-Human Conflict in India.Final Report to the U.S. Fish and Wildlife Service.Asian Nature Conservation Foundation, Bangalore.

[3] Seneviratne, L., Rossel, G., Gunasekera, H. L., Madanayake, Y. M. S. S., \&Doluweera, G. (2004).
Elephant infrasound calls as a method for electronic elephant detection. In Proc. of the Symp. on HumanElephant Relationships and Conflicts (pp. 1-7).

[4] G.D. De Silva, Kasun de Zoysa, (2009). A Low Cost Infrasound Recording System, in 27th National Information Technology Conference, Sri Lanka: The Computer Society of Sri Lanka.

[5] Venter, P. J., \&Hanekom, J. J. (2010). Automatic detection of African elephant (Loxodontaafricana) infrasonic vocalisations from recordings. Biosystems engineering, 106(3), 286-294.

[6] Sugumar.S.J\&Jayaparvathy.R. (2013). An Early Warning System for Elephant Intrusion along the Forest Border Areas. CURRENT SCIENCE. 104. 1515 - 1526.

[7] S. J. Sugumar and R. Jayaparvathy, "An Improved Real Time Image Detection System for Elephant Intrusion along the Forest Border Areas," The Scientific World Journal, Hindawi Publications, vol. 2014, Article ID 393958, 10 pages, 20 January 2014. doi:10.1155/2014/393958

[8] Nair, S., Balakrishnan, R., Seelamantula, C. S., \&Sukumar, R. (2009). Vocalizations of wild Asian elephants (Elephasmaximus): Structural classification and social context. The Journal of the Acoustical Society of America, 126(5), 2768-2778.

[9] Thede, L. D. (2005). Practical analog and digital filter design. New Jersey: Artech House

[10] Stoica, P., \& Moses, R. L. (2005). Spectral analysis of signals. Upper Saddle River, NJ: Pearson/Prentice Hall.

[11] De Cheveigné, A., \& Kawahara, H. (2002). YIN, a fundamental frequency estimator for speech and music. The Journal of the Acoustical Society of America, 111(4), 1917-1930.

[12] Larson, E., \& Maddox, R. (2005). Real-time timedomain pitch tracking using wavelets. Proceedings of the University of Illinois at Urbana Champaign Research Experience for Undergraduates Program.

[13] Pelle, P. A. (2006, May). A robust pitch extraction system based on phase locked loops. In Acoustics, Speech and Signal Processing, 2006. ICASSP 2006 Proceedings. 2006 IEEE International Conference on (Vol. 1, pp. I-I). IEEE.

[14] Ellis, D. P., \& Lee, B. S. (2012). Noise Robust Pitch Tracking by Subband Autocorrelation Classification. In 13th Annual Conference of the International Speech Communication Association.

[15] www.elephantvoices.org

[16] www.coimbatoreforests.org

\section{BIOGRAPHIES}

S.J.Sugumar, has a teaching experience of 15 years. He serves as an Associate Professor in Coimbatore Institute of Technology, Coimbatore currently. $\mathrm{He}$ has been the CoPrincipal Investigator of AICTE sponsored project WSN and project for ElephantIntrusion Detection in the Forest border 
areas done in collaboration with the Forest Department, Tamil Nadu. His areas of interest include Embedded Systems, VLSI and Wireless Sensor Networks.

J.Nirmal Prince, is pursuing M.E in the field of Embedded and Realtime Systems at Coimbatore Institute of Technology. He is a Technical Trainer for Corporates and Colleges for the past 18 months. His areas of interests are Embedded Systems, Wireless Sensor Networks and Web Technology. 